\title{
An Universal and Easy-to-Use Model for the Pressure of Arbitrary-Shape 3D Multifunctional Integumentary Cardiac Membranes
}

\author{
Yewang Su,* Zhuangjian Liu, and Lizhi Xu
}

Tools for cardiac physiological mapping and stimulation are of central interest for understanding, diagnosis, and treatment of cardiac diseases. Although extensive research efforts have been made over the past several decades, ${ }^{[1-4]}$ difficulties remain on establishing conformal, organ-specific physical interfaces between the cardiac structures and advanced electronic hardware. The emerging concepts of stretchable electronics enable devices with physical characteristics matching the natural properties of human tissues, which suggests new routes for addressing these challenges. ${ }^{[5-10]}$ Recently developed stretchable 3D multifunctional integumentary membranes (3D-MIM) enabled a conformal electronic platform that can integrate with the full 3D geometry of the heart, which provides new capabilities for both basic physiological investigation and clinical applications. ${ }^{[11]}$ This class of devices is constructed in a thin, elastic membrane format that matches precisely the 3D geometry of epicardium (Figure 1), with integrated sensors capable of spatiotemporal measurement for electrical activation, temperature, strain, $\mathrm{pH}$, as well as actuators for delivering electrical, thermal, and optical stimulations.

The mechanical compatibility of the 3D-MIM with the beating heart represents a key requirement for the optimum design of the devices. A critical aspect for the compatibility

Prof. Y. Su

State Key Laboratory of Nonlinear Mechanics

Institute of Mechanics

Chinese Academy of Sciences

Beijing 100190, China

E-mail: yewangsu@imech.ac.cn

Prof. Y. Su

Department of Civil and Environmental Engineering

Northwestern University

Evanston, IL 60208, USA

Prof. Z. Liu

Institute of High Performance Computing

A*Star

138632, Singapore

Dr. L. Xu

Department of Chemical Engineering and Department of Materials

Science and Engineering

University of Michigan

Ann Arbor, MI 48109, USA

Dr. L. Xu

Department of Materials Science and Engineering and Frederick Seitz

Materials Research Laboratory

University of Illinois at Urbana-Champaign

Urbana, IL 61801, USA

DOI: 10.1002/adhm.201501029 design is that the 3D-MIM needs to maintain stable contact with the cardiac structures, but with sufficiently low level of pressure on the cardiac muscles to avoid disruption of natural behavior of the heart. It is a difficult task to experimentally measure the pressure exerted by 3D-MIM. The calculation of the pressure by mechanical model is therefore critically important to guide optimum designs for the device. The concept of average pressure is preferred for the quantification, while the distribution of the pressure is actually nonuniform due to the irregular shape of the heart. For simplification, we proposed an analytic model in which expandable ellipsoid is adopted to approximate the beating heart for the estimation of the pressure induced by the surrounding cardiac membrane. ${ }^{[11]}$ That model worked for that specific case, but has two disadvantages: (1) the shape of the heart must approach ellipsoid; (2) it is a difficult task to decide to effective radii of the ellipsoid from the heart with irregular shape, which significantly affects the resulting average pressure. In this paper, a universal and easy-to-use model is established for the calculation of the pressure between the 3D-MIM and the heart with arbitrary shape for various organs, which overcomes these disadvantages.

The 3D-MIM is fabricated with a shrunken size than the systolic state of the heart, ${ }^{[11]}$ so that stable contact can be maintained for both the systolic and diastolic state of the heart. Figure 2 shows a 3D-MIM covering on the expanding heart. The 3D-MIM is modeled as membrane in the mechanical analysis. The membrane force of the cardiac membrane per original unit length, can be obtained according to the theory of membrane as ${ }^{[12]}$

$T_{1}=\frac{E t\left(\varepsilon_{1}+v \varepsilon_{2}\right)}{\left(1-v^{2}\right)}, T_{2}=\frac{E t\left(\varepsilon_{2}+v \varepsilon_{1}\right)}{\left(1-v^{2}\right)}$

where $t$ is the thickness, $E$ and $v$ are the Young's modulus and Poisson's ratio of the membrane, $\varepsilon_{1}$ and $\varepsilon_{2}$ are the membrane strain along the orthorhombic principle directions, respectively. The elastic energy is the integration of the energy density over the entire membrane area ${ }^{[12]}$

$U_{\mathrm{e}}=\iint_{\Omega}\left(\int_{0}^{\varepsilon_{1}} T_{1} \mathrm{~d} \varepsilon_{1}+\int_{0}^{\varepsilon_{2}} T_{2} \mathrm{~d} \varepsilon_{2}\right) \mathrm{d} S$

where $\Omega$ is the surface of the heart. It is reasonable to neglect the effect of the cardiac membrane on the expansion of the heart, since the membrane is very thin and soft. Therefore, the deformation of both the heart and the membrane is free expansion with linear strain 


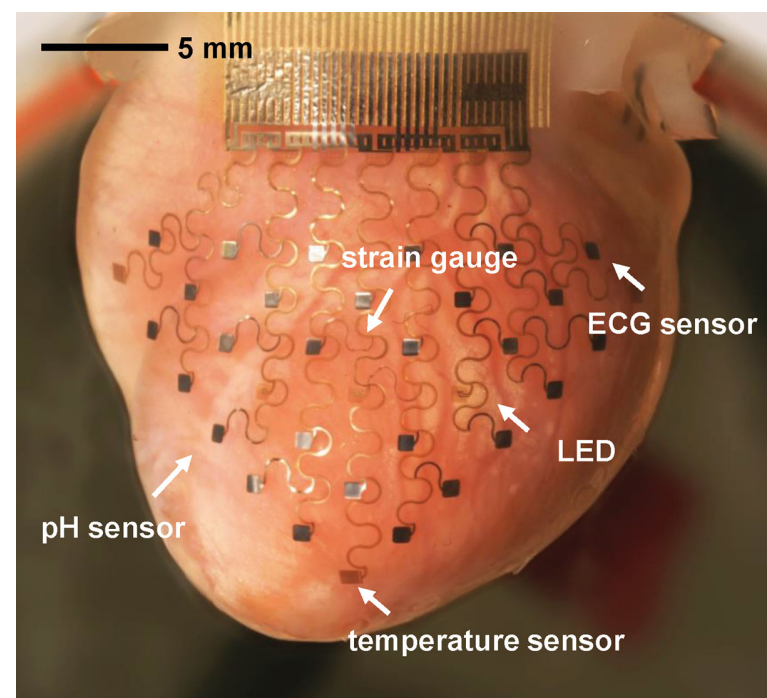

Figure 1. Optical image of a 3D multifunctional integumentary membrane integrated on a Langendorff-perfused rabbit heart.

$\varepsilon_{1}=\varepsilon_{2}=\varepsilon$

The volume and surface area of heart change from $V_{0}$ and $S_{0}$ to $V=(1+\varepsilon)^{3} V_{0}$ and $S=(1+\varepsilon)^{2} S_{0}$ during the expansion, respectively. The membrane force and corresponding elastic energy become $T_{1}=T_{2}=E t \varepsilon /(1-v)$ and $U_{e}=E t \varepsilon^{2} S_{0} /(1-v)$. The average pressure can be obtained as

$P_{\text {average }}=\frac{\mathrm{d} U_{e}}{\mathrm{~d} V}=\frac{2 E t \varepsilon S_{0}}{3(1-v)(1+\varepsilon)^{2} V_{0}}$

according to the principle of energy, i.e., the elastic energy is the work of the pressure on the change of the heart volume. ${ }^{[12]}$

This model must be capable of degeneration to the ideal sphere, since it is for the heart and 3D-MIM with arbitrary shape. For a sphere with radius $R$, the surface area is $S_{0}=4 \pi R^{2}$ and the volume is $V_{0}=4 \pi R^{3} / 3$. Equation (4) gives the average pressure as

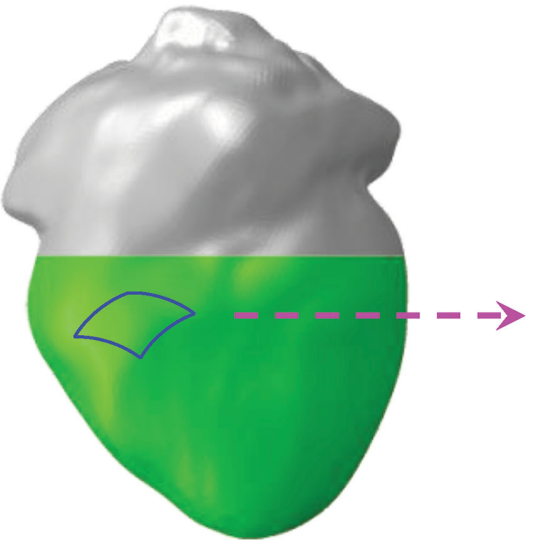

\section{Ecoflex film after expansion}

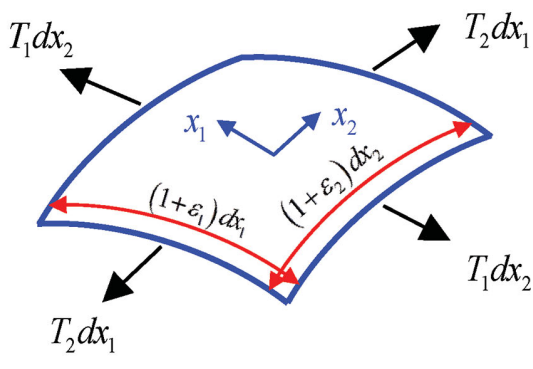

Figure 2. The model of a 3D-MIM covering on the expanding heart and the schematic illustration of the mechanical analysis.

$$
P_{\text {average, sphere }}=\frac{2 E t \varepsilon}{(1-v)(1+\varepsilon)^{2} R}
$$

By the method of equilibrium that is established after expansion, the average pressure can be calculated as

$$
P_{\text {average, sphere }}=\frac{2 \bar{T}}{\bar{R}}
$$

where $\bar{T}=E t \varepsilon /[(1-v)(1+\varepsilon)]$ is the membrane force per unit length after deformation, i.e., accounting for the change of length due to linear expansion, $\bar{R}=(1+\varepsilon) R$ is the radius after expansion. Substitution of $\bar{T}$ and $\bar{R}$ into Equation (6) yields the same expression with Equation (5), which is degenerated from the model with arbitrary shape.

The 3D-MIM covers only the bottom part of the heart with height of about $20.5 \mathrm{~mm}$ in our experiment, instead of the entire body (Figures 1 and 2). In order to use Equation (4), we can imagine a symmetric model consisting of two of this part, with double surface area and volume. Therefore, the surface area should be that of the cardiac membrane, but does not include the area of cross section. For $E=60 \mathrm{kPa}, v=0.49$, $t=150 \mu \mathrm{m}, S_{0}=1303.9 \mathrm{~mm}^{2}$, and $V_{0}=5677.9 \mathrm{~mm}^{3}$ in the experiment, the average pressure are 150 and $278 \mathrm{~Pa}$ for volume expansion $(1+\varepsilon)^{3}-1=20 \%$ (the systolic state of the heart) and $(1+\varepsilon)^{3}-1=45 \%$ (the diastolic state of the heart), respectively. Figure 3 shows the average pressure between the 3D-MIM and the heart for a wide range of device parameters and the expansion of the heart. The average pressure increases with the volume expansion of the heart monotonously and with tensile stiffness Et linearly. The prediction by Equation (4) agrees well with the simulation results by finite element analysis (FEA), even though the detailed shape of the heart and 3D-MIM are rather irregular (see details in following paragraph).

In order to understand the essential mechanical effect of the 3D-MIM on the heart physiology, we can relate this artificial, instrumented membrane to the natural membrane surrounding the heart, i.e., pericardium. In normal physiological states, the pressure within the space created by the pericardium stays at a relatively low level, 0 to a few $\mathrm{mm} \mathrm{Hg}(1 \mathrm{~mm}$ $\mathrm{Hg}=133.3 \mathrm{~Pa})$. In conditions associated with pericardial constraint, which might be caused by fluid accumulation or inflammation, the pericardial pressure increases dramatically (to up to tens of $\mathrm{mm} \mathrm{Hg},>1000 \mathrm{~Pa}$ ). Therefore, the design target for mechanically compatible 3D-MIM is essentially limiting the pressure to the level that is similar to normal pericardial pressure. The present design in our 3D-MIM yields an average pressure $<300 \mathrm{~Pa}$ for diastolic state of the heart according to the analysis. It is sufficiently small to meet the requirement. In practical environment, the 3D-MIM actually provides an additional pressure based on the pressure of the environment, such as from other organs. The two pressures can be considered as superposition. It should be safe since the pressure from 3D-MIM is sufficiently small. 
(a)

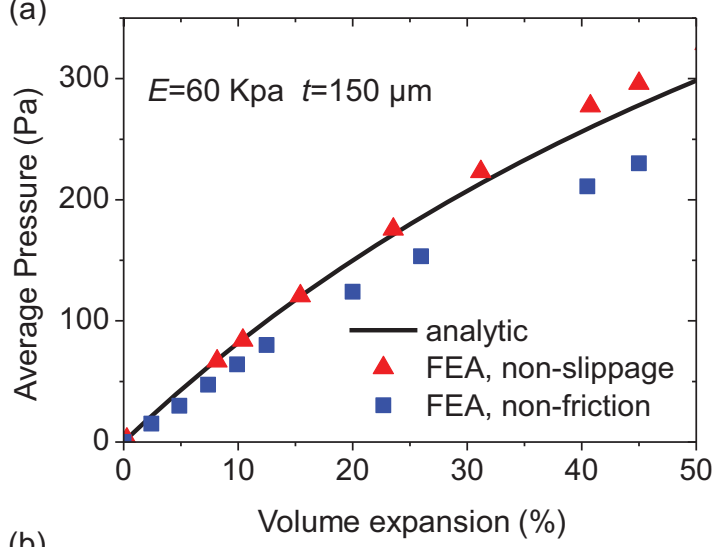

(b)

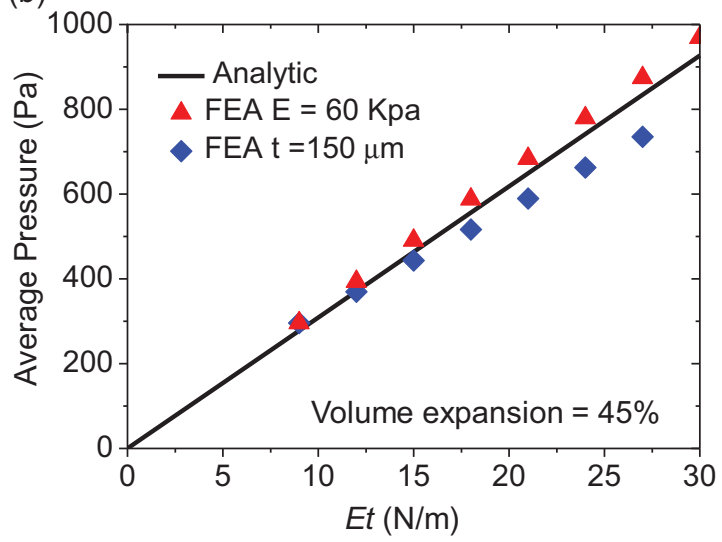

Figure 3. FEA and analytical results of average pressure as functions of a) volume expansion and $b$ ) tensile stiffness $E_{t}$ of the membrane.
Numerical simulation is used to verify the theoretical prediction. The 3D geometric model of the heart for FEA is reconstructed from the data obtained with optical segmentation. The geometric model is imported into the preprocessor in the ABAQUS, a finite element program. ${ }^{[13]}$ heart and 3D-MIM are modeled by the 4-node, linear tetrahedron solid element C3D4 and 4-node quadrilateral membrane element M3D4, respectively. The total number of elements exceeds 60000 , and mesh refinement ensures the accuracy of the numerical results. For the prescribed expansion of the heart, FEA gives the pressure distribution at the interface between the 3D-MIM and the heart. The average pressure is then obtained over the contact area between the 3D-MIM and the heart, i.e., the ventricles of the heart as in the experiment.

The contact condition between the 3D-MIM and the heart is unclear and difficult to verify by experiment. Here, two extreme contact conditions, including nonfriction condition with allowed delamination and nonslippage condition without delamination, are applied in FEA. Figure 3a shows that the difference between the results of the two cases is small and both of them agree with the analytic prediction by Equation (4). The distribution of the pressure of the two conditions is shown in Figure 4 for volume expansion $(1+\varepsilon)^{3}-1=20 \%$ (the systolic state of the heart) and $(1+\varepsilon)^{3}-1=45 \%$ (the diastolic state of the heart), respectively. The local pressure at specific point for the two conditions may be different as shown in Figure 4, but the average pressure over the entire region is similar.

The analysis and simulation above does not account for the effect of electronic devices on the pressure between the 3D-MIM and heart, which may enhance the stiffness of the membrane, as well as the average pressure. This effect can

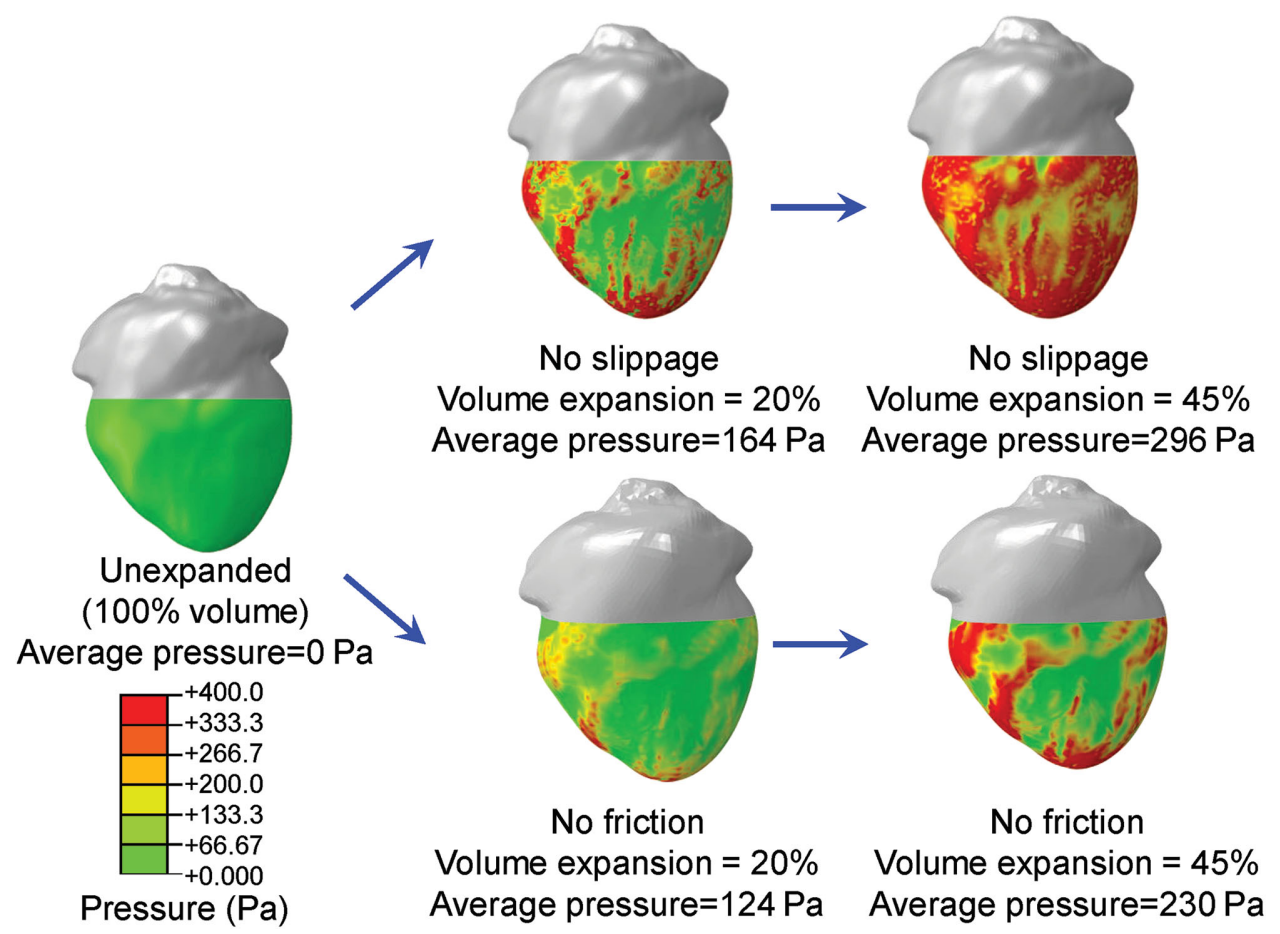

Figure 4. Calculated pressure distribution on the heart induced by 3D-MIM with thickness of $150 \mu \mathrm{m}$ and Young's modulus of $60 \mathrm{kPa}$ under extreme contact conditions and volume expansions. 
be estimated from Equation (4) by replacing the tensile stiffness Et of the 3D-MIM with the effective tensile stiffness of the 3D-MIM with the electronic devices. For the case in our experiment, it is about 1.5 time of present Et without electronic device, according to FEA in our previous work. ${ }^{[11]}$

In summary, we developed a universal and easy-to-use model to calculate the average pressure associated with integration of 3D-MIMs on arbitrary organ shape, which serves as a key parameter to evaluate device designs. The previous model for the prediction of the average pressure was limited in the range of approximated ellipsoid. It is a difficult task to decide the radii of the ellipsoid. Based on the principle of energy, we established a universal and easy-to-use model for the calculation of the pressure of arbitrary-shape 3D-MIM. The average pressure is expressed in terms of only the surface area and volume of the heart, besides the material parameters and expansion strain. The prediction by this new model agrees well with the numerical results by FEA. It is also confirmed by FEA that the average pressure is insensitive to the friction and delamination between the 3D-MIM and the heart. These results provide a simple way to quantify the average pressure for designs of not only 3D-MIM, but also other similar advanced organ-mounted medical devices and optimization.

\section{Acknowledgements}

Y.S. acknowledges the support from Chinese Academy of Sciences via the "Hundred Talent program" and support from NSFC (No. 11572323).

Received: December 18, 2015

Revised: January 4, 2016

Published online: February 18, 2016
[1] L. Harrison, R. E. Ideker, W. M. Smith, G. J. Klein, J. Kasell, A. G. Wallace, J. J. Gallagher, Pacing Clin. Electrophysiol. 1980, 3, 531.

[2] S. J. Worley, R. E. Ideker, J. Mastrototaro, W. M. Smith, H. Vidaillet, P. S. Chen, J. E. Lowe, Pacing Clin. Electrophysiol. 1987, 10, 21.

[3] T. A. Farid, K. Nair, S. Masse, M. A. Azam, A. Maguy, P. F. Lai, K. Umapathy, P. Dorian, V. Chauhan, A. Varro, Circ. Res. 2011, 109, 1309.

[4] O. P. Faris, F. J. Evans, D. B. Ennis, P. A. Helm, J. L. Taylor, A. S. Chesnick, M. A. Guttman, C. Ozturk, E. R. McVeigh, Ann. Biomed. Eng. 2003, 31, 430.

[5] T. Sekitani, Y. Noguchi, K. Hata, T. Fukushima, T. Aida, T. Someya, Science 2008, 321, 1468

[6] M. Kaltenbrunner, T. Sekitanj, J. Reeder, T. Yokota, K. Kuribara, T. Tokuhara, M. Drack, R. Schwodiauer, I. Graz, S. Bauer-Gogonea, Nature 2013, 499, 458.

[7] D. J. Lipomi, M. Vosgueritchian, B. C. Tee, S. L. Hellstrom, J. A. Lee, C. H. Fox, Z. Bao, Nat. Nanotechnol. 2011, 6, 788.

[8] H. Yao, A. J. Shum, M. Cowan, I. Lähdesmäki, B. A. Parviz, Biosens. Bioelectron. 2011, 26, 3290.

[9] D.-H. Kim, N. Lu, R. Mam, Y.-S. Kim, R.-H. Kim, S. Wang, J. Wu, S. M. Won, H. Tao, A. Islam, Science 2011, 333, 838.

[10] D.-H. Kim, N. Lu, R. Ghaffari, Y.-S. Kim, S. P. Lee, L. Xu, J. Wu, R.-H. Kim, J. Song, Z. Liu, Nat. Mater. 2011, 10, 316.

[11] L. Xu, S. R. Gutbrod, A. P. Bonifas, Y. Su, M. S. Sulkin, N. Lu, H.-J. Chung, K.-I. Jang, Z. Liu, M. Ying, C. Lu, R. C. Webb, J.-S. Kim, J. I. Laughner, H. Cheng, Y. Liu, A. Ameen, J.-W. Jeong, G.-T. Kim, Y. Huang, I. R. Efimov, J. A. Rogers, Nat. Commun. 2014, 5, 3329.

[12] S. P. Timoshenko, S. Woinowsky-Krieger, Theory of plates and shells, McGraw-Hill, New York, 1959.

[13] Dassault-Systèmes. Abaqus Analysis User's Manual, v.6.10, Dassault Systèmes Simulia Corp., Rhode Island, 2010. 